\title{
Outage Performance Analysis of Cell-Center/Edge Users Under Two Policies of Energy Harvesting
}

\author{
Dinh-Thuan Do ${ }^{1,2}$, Anh-Tu Le ${ }^{3}$, Chi-Bao Le ${ }^{3}$, Si-Phu Le $^{4}$, Hong-Nhu Nguyen ${ }^{4}$, Lukas Sevcik $^{4}$, \\ Miroslav Voznak ${ }^{4}$ \\ ${ }^{I}$ Wireless Communications Research Group, Ton Duc Thang University, \\ Ho Chi Minh City, Vietnam \\ ${ }^{2}$ Faculty of Electrical \& Electronics Engineering, Ton Duc Thang University, \\ Ho Chi Minh City, Vietnam \\ ${ }^{3}$ Faculty of Electronics Technology, Industrial University of Ho Chi Minh City, \\ Ho Chi Minh City, Vietnam \\ ${ }^{4}$ VSB Technical University of Ostrava, \\ 17. Listopadu St. 2172/15, 70833 Ostrava-Poruba, Czech Republic \\ dodinhthuan@tdtu.edu.vn
}

\begin{abstract}
In this paper, two energy harvesting policies deploying in cooperative non-orthogonal multiple access (NOMA) systems are considered. After period of wireless power transfer, the NOMA users including cell-edge and cell-center users simultaneously transmit the superposition coded symbols to the base station (BS). In the last time slot, the BS decodes to achieve its signal based on superposition coded symbol with corresponding power allocation factors. This paper provides exact expressions of outage probability in two schemes. Performance gap of two NOMA users can be raised by providing different power allocation factors. It is confirmed by numerical result. Distance and data rate are main factors affecting outage performance. Scheme I exhibit scenario where power beacon transmits energy signal to NOMA user while the BS feeds energy to NOMA user in Scheme II. It is shown that outage performance of Scheme I is better than that of Scheme II.
\end{abstract}

Index Terms-Non-orthogonal multiple access; Power beacon; Energy harvesting; Outage performance.

\section{INTRODUCTION}

As a candidate for the $5 \mathrm{G}$ communication, a novel multiple access (MA) technique, named non-orthogonal multiple access (NOMA), has been widely investigated. Main advantages of NOMA include balanced user fairness, its superior spectral efficiency, massive connections, and low access latency [1]-[8]. It is proved that NOMA is introduced to address the spectral efficiency improvement compared with traditional multiple access schemes, i.e., orthogonal multiple

Manuscript received 3 February, 2019; accepted 2 June, 2019.

Part of research of D.-T. Do was supported by Foundation for Science and Technology Development of Ton Duc Thang University (FOSTECT, website: http://fostect.tdt.edu.vn) under Grant FOSTECT.2017.BR.21.

Part of research of M. Voznak was supported by the Ministry of Education, Youth and Sports from the Large Infrastructures for Research, Experimental Development and Innovations project, IT4Innovations National Supercomputing Center - LM2015070“" and partially received a financial support from grant No. SGS SP2019/41 conducted at VSB Technical University of Ostrava, Czech Republic. access (OMA). Up to now, NOMA networks have been explored extensively in various system models. Generally, NOMA networks can be distributed into two classifications by employing spreading signature of MA: code-power NOMA (CD NOMA) and power-domain NOMA (PD NOMA). In contrast to frequency-division multiple access (FDMA) and time-division multiple access (TDMA) in conventional orthogonal multiple access (OMA) [9], NOMA examines the non-orthogonal resource distribution. That is how the key idea of NOMA to realize MA the power domain is exploited. As a result, different power levels are allocated to distinguished users at transmitter side [10]-[12]. At receiver side, to cancel the multiuser interference at the receiver, the successive interference cancellation (SIC) is assigned [13]. More specifically, the NOMA user associated with a worse channel condition needs to be allocated with higher power.

In addition, dangers related to batteries leakage and many of these energy-limited applications using batteries that cannot be substituted or re-energized are explored. Fortunately, recent research interest introduced energy harvesting as only one solution to tackle such a problem, though energy harvesting is that has generated a great deal applied in wireless sensor networks [14]-[18]. Compared to using traditional batteries in terms of batteries replacement and batteries external recharging, energy harvesting can exhibit essentially limitless energy. Therefore, to enhance spectrum efficiency and prolong the lifetime of the system, NOMA networks with energy-limited devices need to employ the efficient integration of energy harvesting with NOMA [19]-[21]. However, malicious energy harvesting receivers (EHRs) may exist and intercept the confidential transmitted information signals. As a result, security of energy harvesting-enabled NOMA systems need to be investigated due to the broadcasting nature of NOMA and RF signals [22]-[25]. The question is how to enlarge the amount of 
harvested energy in such NOMA networks. Motivated by interesting results in [1], we develop two scenarios of energy harvesting to show different performance improvements in NOMA.

The reminder of our research work is organized as follows. The system model and policies of energy harvesting are presented in Section II. The exact outage probability of two schemes are provided in Section III and Section IV, and Section V presents simulation results. The paper concludes with Section VI.

\section{System Model AND ENERGy HARVESTING POLICIES IN NOMA}

We consider new system model, as it is shown in Fig.1 and Fig. 2, containing three nodes (the base station denoted as BS, two NOMA users $U_{1}$ and $U_{2}$ ), which are deployed to serve two transmission scenarios in NOMA system. Regarding energy harvesting, energy harvesting policy with the specific scheme of Time Switching (TS)-based energy policy performs wireless charge to cell-edge user 1 and cell-center user 2 (i.e., $U_{1}, U_{2}$ ) (Fig.1 and Fig. 2). In Fig. 2, only cell-edge user $U_{1}$ needs charge by wireless energy transfer. It is noted that TS is selected due to simpler hardware implementation in practice. In this model, a single-antenna half-duplex node is assumed at each node. The distance of link BS- $U_{1}$ and BS- $U_{2}$ are $d_{a}, d_{b}$ respectively, where $d_{b}>$ $d_{a}$ and these links follow quasi static-Rayleigh channel model, and the path loss exponent is denoted by $m$. We call $h_{a}$ and $h_{b}$ as coefficients between BS and $U_{1}$ and BS and $U_{2}$, respectively. As there are many works in literature, these channels are assumed as independent components and they have zero mean and unit variance. Next, the BS has transmit power as $P$. In NOMA scheme, $x_{a}$ and $x_{b}$ are separated messages, which are sent by NOMA user 1 and NOMA user 2 , respectively, in uplink for the first scenario. At each user, $U_{1}$ and $U_{2}$, noise terms are denoted as $n_{a}, n_{b}$ following $C N\left(0, \sigma^{2}\right)$. They are the Additive White Gaussian Noise (AWGN) with equal variance $\sigma^{2}$.

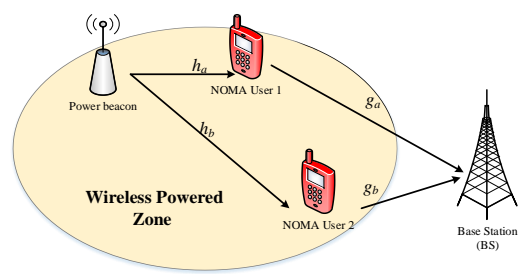

Fig. 1. Scheme I: Uplink NOMA with wireless power transfer from beacon.

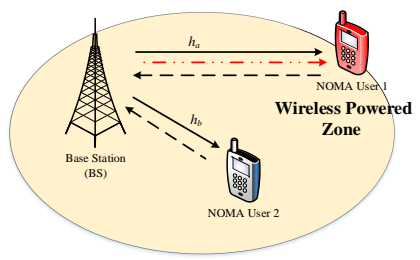

Fig. 2. Scheme II: Hybrid downlink-uplink NOMA with wireless power transfer from the base station.
This study considers two scenarios:

- - SCHEME I: Power beacon charges energy to different NOMA users. Such NOMA users deploy harvested energy to transmit signal in uplink to the BS;

- - SCHEME II: If there is not any help of power beacon, the BS transmits signal in downlink to two users in the first phase, then the BS charges energy to two users in the second phase. At the third phase, users intend to transmit signal in uplink to the BS. Scheme II is similar model with detailed presentation as in [1].

\section{SCHEME I: POWER BEACON-BASED ENERGY HARVESTING NOMA}

\section{A. First Phase: Energy Harvesting}

To serve signal transmission in following epoch, the NOMA user can be able to harvest energy from the power beacon. Such technique enables wireless power charge for both NOMA users. By denoting $x_{e}$ as energy-bearing signal at NOMA user 1 , we obtain the received signal as

$$
y_{U_{1}, E H}=\frac{\sqrt{P} x_{e} h_{a}}{\sqrt{d_{a}^{m}}}+n_{a} .
$$

Similarly, the received signal for energy collection at NOMA user 2 is expressed by

$$
y_{U_{2}, E H}=\frac{\sqrt{P} x_{e} h_{b}}{\sqrt{d_{b}^{m}}}+n_{b} .
$$

Regarding to wireless power transfer scheme, time allocation for energy harvesting phase is $T / 2$ and the amount of harvested energy is computed by

$$
E_{h}^{T}=\left(\frac{\eta P\left|h_{a}\right|^{2}}{d_{a}^{m}}+\frac{\eta P\left|h_{b}\right|^{2}}{d_{b}^{m}}\right) \frac{T}{2}
$$

Following the principle of energy harvesting [14-19], the harvested power is given by

$$
P_{a, E H}=\frac{\eta P\left|h_{a}\right|^{2}}{d_{a}^{m}},
$$

where $\eta$ is the energy conversion efficiency and it depends on hardware circuits $(0<\eta<1)$. Similarly, the energy harvested at $U_{2}$ is given as follows

$$
P_{b, E H}=\frac{\eta P\left|h_{b}\right|^{2}}{d_{b}^{m}}
$$

Remark 1: It is noted that the amount of harvested energy at $U_{1}$ is higher than that of $U_{2}$ because of different distances to the power beacon, which result in better channel power gain.

\section{B. Second Phase: Uplink NOMA}

Next, we deploy uplink for NOMA transmission in the 
second phase using harvested power for signal transmission. By using harvested power, $P_{\mathrm{a}, E H}$ and $P_{b, E H}$ are employed to transmit signal in the uplink, respectively. The received NOMA signal can be obtained as

$$
y_{U}=\frac{\sqrt{P_{a, E H}} x_{a}^{\prime} g_{a}}{\sqrt{d_{a}^{m}}}+\frac{\sqrt{P_{b, E H}} x_{b}^{\prime} g_{b}}{\sqrt{d_{b}^{m}}}+n_{S},
$$

where $n_{S}$ follows $C N \in\left(0 ; \sigma^{2}\right)$ and it denotes the AWGN at the BS with variance $\sigma^{2}$ while $x_{a}^{\prime}$ and $x_{b}^{\prime}$ are the uplink signal from $U_{1}$ and $U_{2}$ respectively.

In this case of NOMA, SIC is performed at BS to identify individual user's message. In particular, considered signals ( $x_{a}^{\prime}$ and $x_{b}^{\prime}$ ) are decoded and decoding order. Such decoding operation is performed by distinguishing different received power levels. It is worth noting that the message with $\left(P_{\mathrm{a}, E H}\right)$ is decoded first as it has the higher power, then the message with lower power $\left(P_{b, E H}\right)$ is decoded. Therefore, the BS has signal to interference plus noise ratio (SINR) and it is given as

$$
\gamma_{U 1}^{x_{a}^{\prime}}=\frac{\left|g_{a}\right|^{2} P_{a, E H}}{d_{a}^{m}\left(\left|g_{b}\right|^{2} P_{b, E H} d_{b}^{-m}+\sigma^{2}\right)} .
$$

By replacing $P_{\mathrm{a}, E H}$ and $P_{b, E H}$ from (3) and (4) in above equation, new expression is

$$
\gamma_{U 1}^{x_{a}^{\prime}}=\frac{\eta \rho\left|h_{a}\right|^{2}\left|g_{a}\right|^{2}}{\eta \rho\left|h_{b}\right|^{2}\left|g_{b}\right|^{2} d_{a}^{2 m} d_{b}^{-2 m}+d_{a}^{2 m}} .
$$

Then, the BS subtracts noise term from the received signal after success decoding $x_{a}^{\prime}$. Next, it continues to decode $x_{b}^{\prime}$. Similarly, to decode $x_{b}^{\prime}$, we compute the SINR at BS such as

$$
\gamma_{U 2}^{x_{b}^{\prime}}=\frac{\left|g_{b}\right|^{2} p_{b}^{\prime}}{\sigma^{2} d_{b}^{2 m}}
$$

As a result, it is transcribed as

$$
\gamma_{U 2}^{x_{b}^{\prime}}=\frac{\eta \rho\left|h_{b}\right|^{2}\left|g_{b}\right|^{2}}{d_{b}^{2 m}} .
$$

\section{Outage Performance Analysis in Scheme I}

In this section, the outage probability is main metric considered in our proposed model. The outage performance is defined in many works [14-17]. We now consider outage probability for such NOMA transmission as below.

We first compute outage probability at the BS for $x_{a}^{\prime}$ and it can be expressed as below.

Firstly, outage event of $x_{a}^{\prime}$ is defined by

$$
O P_{S}^{x_{a}^{\prime}}=1-\operatorname{Pr}\left(\gamma_{U_{1}}^{x_{a}^{\prime}}>\varphi_{U L}^{U_{1}}\right)
$$

It can be re-expressed by

$$
O P_{S}^{x_{a}^{\prime}}=\operatorname{Pr}\left(\frac{\frac{\eta \rho\left|h_{a}\right|^{2}\left|g_{a}\right|^{2}}{d_{a}^{2 m}}}{\frac{\eta \rho\left|h_{b}\right|^{2}\left|g_{b}\right|^{2}}{d_{b}^{2 m}}+1}<\varphi_{U L}^{U_{1}}\right) .
$$

Interestingly, by considering exponential distribution for $\left|h_{a}\right|^{2}$ and $\left|h_{b}\right|^{2}$ with respect to integration variables $x, y$, the Cumulative Distribution Function (CDF) and Probability Density Function (PDF) of $\left|h_{a}\right|^{2}\left|g_{a}\right|^{2}$ are defined as follows:

$$
\begin{gathered}
F_{\frac{\eta \rho\left|h_{a}\right|^{2}\left|g_{a}\right|^{2}}{d_{a}^{2 m}}}(x)=1-2 \sqrt{\frac{x}{\lambda_{a}}} K_{1}\left(2 \sqrt{\frac{x}{\lambda_{a}}}\right), \\
f_{\frac{\eta \rho\left|h_{b}\right|^{2}\left|g_{b}\right|^{2}}{d_{b}^{2 m}}}(x)=\frac{2}{\lambda_{b}} K_{0}\left(2 \sqrt{\frac{x}{\lambda_{b}}}\right),
\end{gathered}
$$

where $\lambda_{a}=\frac{\eta \rho}{d_{a}^{2 m}}, \quad \lambda_{b}=\frac{\eta \rho}{d_{b}^{2 m}}$, and $\varphi_{U L}^{U_{1}}=2^{R_{1}^{U L}}-1$ is the required threshold SINR to decode related singal at BS, i.e., $x_{a}^{\prime}$ sent by $U_{1}$. Hence, we can further simplify $O P_{S}^{x_{a}^{\prime}}$ as follows

$$
\begin{aligned}
& O P_{S}^{x_{a}^{\prime}}=1-\frac{2}{\lambda_{b}} \int_{0}^{\infty} \frac{2 \sqrt{\varphi_{U L}^{U_{1}}}}{\sqrt{\lambda_{a}}} \sqrt{z+1} \times \\
& \times K_{1}\left(\frac{2 \sqrt{\varphi_{U L}^{U_{1}}}}{\sqrt{\lambda_{a}}} \sqrt{z+1}\right) K_{0}\left(\frac{2}{\sqrt{\lambda_{b}}} \sqrt{z}\right) d z,
\end{aligned}
$$

where $K_{1}($.$) is the Bessel function of first order second kind.$

Then, the outage performance at the BS for $x_{b}^{\prime}$ can be computed as below.

Similarly, outage event of $x_{b}^{\prime}$ is computed as

$$
\begin{gathered}
O P_{U_{1}}^{x_{b}^{\prime}}=1-\operatorname{Pr}\left(\gamma_{U_{1}}^{x_{a}^{\prime}}>\varphi_{U L}^{U_{1}}, \gamma_{U_{2}}^{x_{b}^{\prime}}>\varphi_{U L}^{U_{2}}\right)= \\
=1-\operatorname{Pr}\left(\frac{\eta \rho\left|h_{a}\right|^{2}\left|g_{a}\right|^{2}}{\eta \rho\left|h_{b}\right|^{2}\left|g_{b}\right|^{2} d_{a}^{2 m} d_{b}^{-2 m}+d_{a}^{2 m}}>\varphi_{U L}^{U_{1}}\right) \times \\
\times \underbrace{\operatorname{Pr}\left(\frac{\eta \rho\left|h_{b}\right|^{2}\left|g_{b}\right|^{2}}{d_{b}^{2 m}}>\varphi_{U L}^{U_{2}}\right)}_{V_{1}} .
\end{gathered}
$$

It has to be noted that the successful decoding of $x_{a}^{\prime}$ results in computing the outage at BS for $x_{b}^{\prime}$. If we denote $\varphi_{U L}^{U_{2}}=2^{R_{2}^{U L}}-1$ as the required threshold SINR to decode $x_{b}^{\prime}$ at BS, then it can be achieved following the proposition.

Proposition 1: The exact expression of outage probability at $\mathrm{BS}$ for $x_{b}^{\prime}$ is given by

$$
O P_{U_{1}}^{x_{b}^{\prime}}=1-\frac{4 \sqrt{\vartheta} K_{1}(2 \sqrt{\vartheta})}{\lambda_{b}} \int_{0}^{\infty} \frac{2 \sqrt{\varphi_{U L}^{U_{1}}}}{\sqrt{\lambda_{a}}} \sqrt{z+1} \times
$$




$$
\times K_{1}\left(\frac{2 \sqrt{\varphi_{U L}^{U_{1}}}}{\sqrt{\lambda_{a}}} \sqrt{z+1}\right) K_{0}\left(\frac{2}{\sqrt{\lambda_{b}}} \sqrt{z}\right) d z
$$

where $\vartheta=\frac{\varphi_{U L}^{U_{2}} d_{b}^{2 m}}{\eta \rho}$.

Proof: Initially, $V_{1}$ can be expressed as

$$
V_{1}=\operatorname{Pr}\left(\left|h_{b}\right|^{2}\left|g_{b}\right|^{2}>\vartheta\right)
$$

where $\vartheta=\frac{\varphi_{U L}^{U_{2}} d_{b}^{2 m}}{\eta \rho}$. Characterizing $\left|h_{b}\right|^{2}$ by exponential distribution, it is required to compute

$$
V_{1}=\operatorname{Pr}\left(\left|h_{b}\right|^{2}\left|g_{b}\right|^{2}>\vartheta\right)
$$

In particular, we have the following expression

$$
V_{1}=\int_{0}^{\infty}\left(e^{-\frac{y}{x}}\right) e^{x} d x
$$

Therefore, new result is given by

$$
V_{1}=\sqrt{4 v} K_{1}(\sqrt{4 \vartheta})
$$

Plugging (15) and (21), we obtain expected result. It completes the proof.

\section{Scheme II: Single Source HARVESTING NOMA}

To enable the hybrid NOMA employing SWIPT, three equal time slots belonging to the whole transmission block $T$ are divided. In this situation, three phases are deployed, i.e., signal transmission in the first phase, energy harvesting in the second phase, and signal transmission in the third phase as well. Based on results of [1], we recall following computations. It is noted that there is only NOMA user 1, who can harvest energy from the BS while NOMA user 2 has the grid power.

\section{A. Uplink NOMA}

In this situation, the uplink NOMA signal received by BS is given as

$$
y_{S}=\frac{\sqrt{P_{a, E H}} x_{a}^{\prime} h_{a}}{\sqrt{d_{a}^{m}}}+\frac{\sqrt{P_{b}} x_{b}^{\prime} h_{b}}{\sqrt{d_{b}^{m}}}+n_{S} .
$$

Then, SINR at the BS is computed to decode $x_{a}^{\prime}$ and it is given as

$$
\gamma_{1}^{x_{a}^{\prime}}=\frac{\left|h_{a}\right|^{2} \rho_{a}^{\prime}}{d_{a}^{m}\left(\left|h_{b}\right|^{2} d_{b}^{-m}+\sigma^{2}\right)} .
$$

The noise term is small. Hence, the energy harvested by NOMA user 1 is computed as follows

$$
\rho_{a}^{\prime}=\frac{\eta P\left|h_{a}\right|^{2}}{d_{a}^{m}}
$$

By substituting $\rho_{a}^{\prime}$ from (6), we obtain

$$
\gamma_{1}^{x_{a}^{\prime}}=\frac{\rho \eta\left|h_{a}\right|^{4}}{\rho\left|h_{b}\right|^{2} d_{a}^{2 m} d_{b}^{-m}+d_{a}^{2 m}} .
$$

In this case, when $x_{a}^{\prime}$ is decoded successfully, the BS subtracts it from the received uplink signal and continues to detect $x_{b}^{\prime}$. Hence, the SINR at the BS to detect $x_{b}^{\prime}$ is formulated by

$$
\gamma_{2}^{x_{b}^{\prime}}=\frac{\rho\left|h_{b}\right|^{2}}{d_{b}^{2 m}}
$$

\section{B. Outage Probability for Uplink NOMA Transmission in Scheme II}

We first consider evaluation at the BS for $x_{a}^{\prime}$ and the related outage probability can be expressed as

$$
\begin{gathered}
O P_{S}^{x_{a}^{\prime}}=1-\operatorname{Pr}\left(\gamma_{1}^{x_{a}^{\prime}}>\varphi_{U L}^{U E_{A}}\right)= \\
=1-\operatorname{Pr}\left(\frac{\eta \rho d_{b}^{m}\left|h_{a}\right|^{4}}{\rho d_{a}^{2 m}\left|h_{b}\right|^{2}+d_{a}^{2 m} d_{b}^{m}}>\varphi_{U L}^{U E_{A}}\right)= \\
=1-\underbrace{\operatorname{Pr}\left(\left|h_{b}\right|^{2} \leq \theta_{1}^{x_{a}^{\prime}}\left|h_{a}\right|^{4}-\theta_{2}^{x_{a}^{\prime}}\right)}_{I_{1}},
\end{gathered}
$$

where $\theta_{1}^{x_{a}^{\prime}}=\frac{\eta d_{b}^{m}}{\varphi_{U L}^{U_{1}} d_{a}^{2 m}}, \quad \theta_{2}^{x_{a}^{\prime}}=\frac{d_{b}^{m}}{\rho}$ and the threshold SINR required at the BS is denoted as $\varphi_{U L}^{U_{1}}=2^{R_{1}^{U L}}-1$ to decode $x_{a}^{\prime}$ sent by $U_{1}$. Hence, we can further simplify $I_{1}$ as follows:

$$
\begin{gathered}
I_{1}=\int_{\sqrt{\chi}}^{\infty}\left(1-e^{\left(\frac{d_{b}^{m}}{\rho}-\delta x^{2}\right)}\right) e^{-x} d x, \\
I_{1}=\underbrace{\int_{\sqrt{\chi}}^{\infty} e^{-x} d x}_{\Upsilon_{1}}-\underbrace{\int_{\chi}^{\infty} e^{\frac{d_{b}^{m}}{\rho}} e^{-\delta x^{2}-x} d x}_{\Upsilon_{2}},
\end{gathered}
$$

where $\chi=\frac{\varphi_{U L}^{U E_{A}} d_{a}^{2 m}}{\eta \rho}$ and $\delta=\frac{\eta d_{b}^{m}}{\varphi_{U L}^{U E_{A}} d_{a}^{2 m}}$, applying [3.322.1, [26]], we can express $\Upsilon_{2}$ as:

$$
\Upsilon_{2}=e^{\frac{d_{b}^{m}}{\rho}} \sqrt{\pi \beta} e^{\beta}\left(1-\Phi\left(\sqrt{\beta}+\frac{\sqrt{\chi}}{2 \sqrt{\beta}}\right)\right)
$$

where $\beta=\frac{1}{4 \delta}$ and $\Phi($.$) is applying [3.322.1, [26]]. By$ solving $r_{1}$ and replacing results of $r_{1}$ and $r_{2}$ in (29) we 
obtain

$$
I_{1}=e^{-\sqrt{\chi}}-e^{\frac{d_{b}^{m}}{\rho}} \sqrt{\pi \beta} e^{\beta}\left(1-\Phi\left(\sqrt{\beta}+\frac{\sqrt{\chi}}{2 \sqrt{\beta}}\right)\right) .
$$

Therefore, $O P_{S}^{x_{a}^{\prime}}$ can be obtained by replacing (31) into (27)

$$
O P_{S}^{x_{a}^{\prime}}=1-e^{-\sqrt{\chi}}+e^{\frac{d_{b}^{m}}{\rho}} \sqrt{\pi \beta} e^{\beta}\left(1-\Phi\left(\sqrt{\beta}+\frac{\sqrt{\chi}}{2 \sqrt{\beta}}\right)\right)
$$

Then, we further consider outage performance of $x_{b}^{\prime}$ as below.

Proposition 2: The outage probability at the BS for $x_{b}^{\prime}$ in uplink transmission in energy harvesting-enabled NOMA is given by

$$
\begin{gathered}
O P_{U_{1}}^{x_{b}^{\prime}}=1-\left(e ^ { - \kappa } \left(e^{-\sqrt{\chi}}+e^{\frac{d_{b}^{m}}{\rho}} \sqrt{\pi \beta} e^{\beta},\right.\right. \\
\left.\left.\left(1-\Phi\left(\sqrt{\beta}+\frac{\sqrt{\chi}}{2 \sqrt{\beta}}\right)\right)\right)\right) .
\end{gathered}
$$

Proof: See the appendix in Section VI.

\section{NUMERICAL AND SIMULATION RESULTS}

The numerical analysis is implemented to evaluate the performance of our proposed system. Simulated results confirm the exactness of the expression derived. It is worth noting that following curves in figures are predicted so that they match with simulation results in all of the results. The distances between pair of nodes are normalized. In this section, $m=3$ is set for the path loss exponent. We set simulations for 10,0000 times.

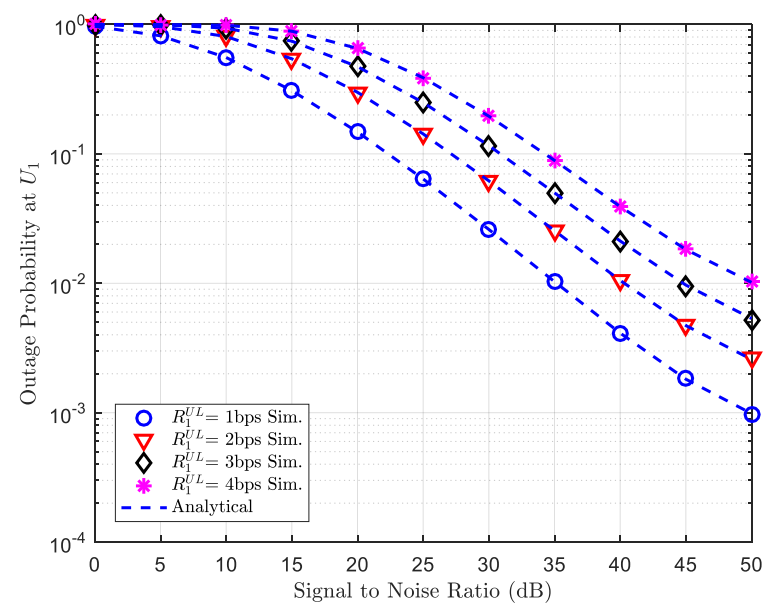

Fig. 3. Outage performance at $U_{1}$ with varying target rate and $d_{a}=1$, $d_{b}=5$, and $\eta=0.2$ in Scheme I.

Fig. 3 and Fig. 4 plot the outage probability for $U_{1}$ and $U_{2}$ as varying SNR in considered range in uplink NOMA transmission. Higher data rates are required, outage performance decreases slightly. The performance gap is visible in the cases when different data rate is small. It exhibits the acceptance of the performance for such NOMA. In addition, power beacon provides enough energy to signal processing at the BS. Powering energy from power beacon is reasonable solution for small devices, which need amount of power for their operation.

Figure 5 clearly shows the important performance of $U_{1}$, where energy harvesting policy is activated. It is worth noting that NOMA user 1 needs harvested energy, which results in worse performance. That is the main reason to consider only the performance of $U_{1}$ as it is shown in Fig. 5. illustration. Our observation results show that at a very low data rate the outage performance of $U_{1}$ is low.

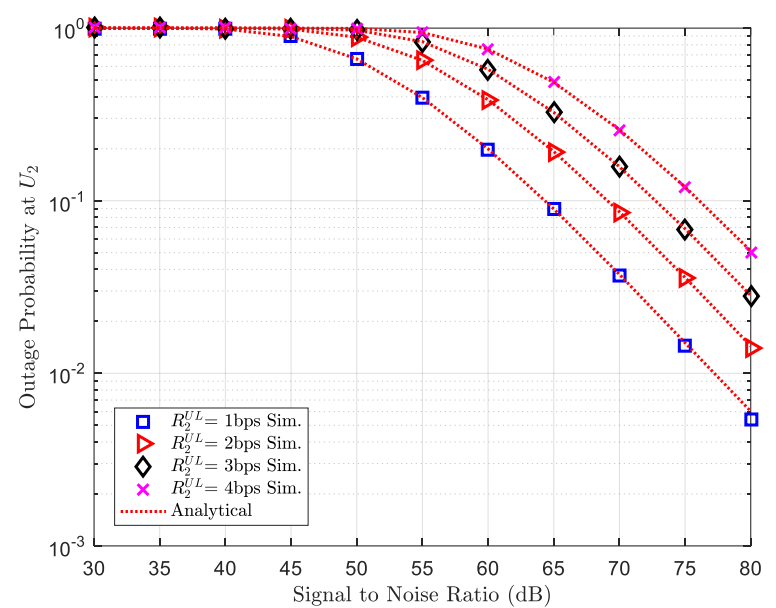

Fig. 4. Outage performance of $U_{2}$ with varying target rate and $d_{a}=1$, $d_{b}=5, R_{1}^{U L}=1, a_{1}=0.1, a_{2}=0.9$, and $\eta=0.2$ in Scheme I.

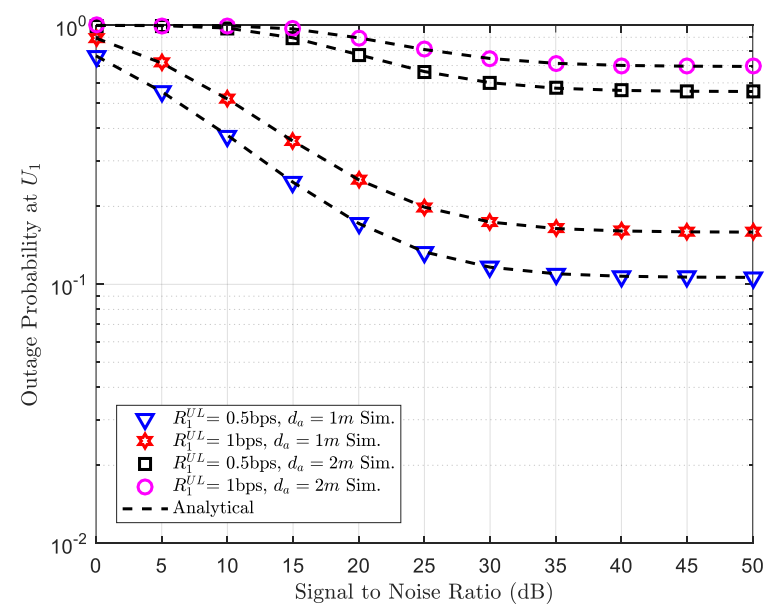

Fig. 5. Outage performance of $U_{1}$ in Scheme II ( $d_{a}=1, d_{b}=5, \eta=0.2$ ).

\section{CONCLUSIONS}

In this paper, in order to remain operation together with acceptable outage performance of NOMA, energy harvesting policies in it are required to be deployed. Two scenarios are proposed, based on which source the energy is transmitted, i.e., the base station or the power beacon. The exact expressions of outage performance are studied under a linear energy harvesting model. As main result, two schemes are 
proposed to solve this challenging wireless power transfer to NOMA users. It is shown that the performance achieved by using NOMA in Scheme I is better than that in Scheme II, although Scheme I requires the power beacon to be designed.

\section{APPENDIX A}

Proof of proposition 2. We further examine the outage event at the BS for $x_{b}^{\prime}$ and it can be formulated by

$$
\begin{gathered}
O P_{U_{1}}^{x_{b}^{\prime}}=1-\operatorname{Pr}\left(\gamma_{1}^{x_{a}^{\prime}}>\varphi_{U L}^{U E_{A}}, \gamma_{2}^{x_{b}^{\prime}}>\varphi_{U L}^{U_{2}}\right)= \\
=1-\underbrace{\operatorname{Pr}\left(\gamma_{1}^{x_{a}^{\prime}}>\varphi_{U L}^{U E_{A}}\right)}_{I_{1}} \underbrace{\operatorname{Pr}\left(\gamma_{2}^{x_{b}^{\prime}}>\varphi_{U L}^{U_{2}}\right)}_{I_{2}} .
\end{gathered}
$$

Next, $I_{2}$ can be expressed as

$$
I_{2}=\operatorname{Pr}\left(\left|h_{b}\right|^{2}>\kappa\right)
$$

where $\kappa=\frac{\varphi_{U L}^{U_{2}} d_{b}^{2 m}}{\rho}$. Then, $I_{2}$ can be computed by using exponential distribution of $\left|h_{b}\right|^{2}$ as

$$
I_{2}=e^{-\kappa}
$$

In the end, it can be obtained final result. It completes the proof.

\section{REFERENCES}

[1] S. K. Zaidi, S. F. Hasan, and X. Gui, "SWIPT-aided uplink in hybrid non-orthogonal multiple access", in Proc. of 2018 IEEE Wireless Communications and Networking Conference (WCNC), Dec. 2018. DOI: 10.1109/WCNC.2018.8376963.

[2] Z. Ding, M. Peng, and H. V. Poor, "Cooperative non-orthogonal multiple access in 5G systems", IEEE Commun. Lett., vol. 19, no. 8, pp. 1462-1465, 2015. DOI: 10.1109/LCOMM.2015.2441064.

[3] D.-T. Do, M.-S. van Nguyen, T.-A. Hoang, and M. Voznak, "NOMA-assisted multiple access scheme for IoT deployment: Relay selection model and secrecy performance improvement", Sensors, vol. 19, no. 3, pp. 736, 2019. DOI: 10.3390/s19030736.

[4] Y. Zhang, H.-M. Wang, and T. Zheng, "Energy-efficient transmission design in non-orthogonal multiple access", IEEE Trans. Veh. Technol. vol. 66 , no. 3 , pp. $2582-2587$, 2016. DOI: 10.1109/TVT.2016.2578949.

[5] V.-D. Nguyen, H. D. Tuan, T. Q. Duong, H. V. Poor, and O.-S. Shin, "Precoder design for signal superposition in MIMO-NOMA multicell networks", IEEE J. Sel. Areas Commun., vol. 35, no. 12 , pp. 2681-2695, 2017. DOI: 10.1109/JSAC.2017.2726007.

[6] T.-L. Nguyen and D.-T. Do, "Exploiting impacts of intercell interference on SWIPT-assisted non-orthogonal multiple access", Wireless Communications and Mobile Computing, vol. 2018, 2018. DOI: 10.1155/2018/2525492.

[7] Z. Ding, P. Fan, and H. V. Poor, "Impact of user pairing on 5G non-orthogonal multiple-access downlink transmissions", IEEE Trans. Veh. Technol., vol. 65, no. 8, pp. 6010-6023, 2016. DOI: 10.1109/TVT.2015.2480766.

[8] N. Zhang, J. Wang, G. Kang, and Y. Liu, "Uplink nonorthogonal multiple access in 5G systems", IEEE Commun. Lett., vol. 20, no. 3, pp. 458-461, 2016. doi:10.1109/LCOMM.2016.2521374.

[9] K. Tourki, H.-C. Yang, and M.-S. Alouini, “Accurate outage analysis of incremental decode-and-forward opportunistic relaying", IEEE Trans. Wireless Commun., vol. 10, no. 4, pp. 1021-1025, 2011. DOI: 10.1109/TWC.2011.021611.100472.

[10] Z. Wei, D. K. Ng, J. Yuan, and H.-M. Wang, "Optimal resource allocation for power-efficient MC-NOMA with imperfect channel state information", IEEE Trans. Commun., vol. 65, no. 9, pp. 3944-3961, 2017. DOI: 10.1109/TCOMM.2017.2709301.

[11] Z. Yang, Z. Ding, P. Fan, and N. Al-Dhahir, "The impact of power allocation on cooperative non-orthogonal multiple access networks with SWIPT", IEEE Trans. Wireless Commun., vol. 16, no. 7, pp. 4332-4343, 2017. DOI: 10.1109/TWC.2017.2697380.

[12] Z. Ding, F. Adachi, and H. V. Poor, "The application of MIMO to non-orthogonal multiple access", IEEE Trans. Wireless Commun., vol. 15, no. 1, pp. 537-552, 2016. DOI: 10.1109/TWC.2015.2475746.

[13] J. Wang, Q. Peng, Y. Huang, H.-M. Wang, and X. You, "Convexity of weighted sum rate maximization in NOMA systems", IEEE Commun. Lett., vol. 24, no. 9, pp. 1323-1326, 2017. DOI: 10.1109/LSP.2017.2722546.

[14] D.-T. Do, H.-S. Nguyen, M Voznak, and T.-S. Nguyen, "Wireless powered relaying networks under imperfect channel state information: system performance and optimal policy for instantaneous rate", Radioengineering, vol. 26, no. 3, pp. 869-877, 2017. DOI: 10.13164/re.2017.0869.

[15] X.-X. Nguyen, D.-T. Do, "Maximum harvested energy policy in full-duplex relaying networks with SWIPT", International Journal of Communication Systems (Wiley), vol. 30, no. 17, 2017. DOI: 10.1002/dac. 3359

[16] X.-X. Nguyen and D.-T. Do, "Optimal power allocation and throughput performance of full-duplex DF relaying networks with wireless power transfer-aware channel", EURASIP Journal on Wireless Communications and Networking, vol. 152, 2017. DOI: 10.1186/s13638-017-0936-X.

[17] T.-L. Nguyen and D.-T. Do, "A new look at AF two-way relaying networks: energy harvesting architecture and impact of co-channel interference", Annals of Telecommunications, vol. 72, no. 11, pp. 669-678, 2017. doi:10.1007/s12243-017-0590-7.

[18] K. T. Nguyen, D.-T. Do, X. X. Nguyen, N. T. Nguyen, and D. H. Ha, "Wireless information and power transfer for full duplex relaying networks: performance analysis", in Proc. of Recent Advances in Electrical Engineering and Related Sciences (AETA 2015), HCMC, Vietnam, pp. 53-62, 2015. DOI: 10.1007/978-3-319-27247-4_5.

[19] D.-T. Do and Ch.-B. Le, "Application of NOMA in wireless system with wireless power transfer scheme: Outage and ergodic capacity performance analysis", Sensors, vol. 18, no. 10, pp. 3501, 2018. DOI: 10.3390/s18103501

[20] Y. Liu, Z. Ding, M. Elkashlan, and H. V. Poor, "Cooperative non-orthogonal multiple access with simultaneous wireless information and power transfer", IEEE J. Select. Areas Commun., vol. 34, no. 4, pp. 938-953, 2016. DOI: 10.1109/JSAC.2016.2549378.

[21] Y. $\mathrm{Xu}$, et al., "Joint beamforming and power-splitting control in downlink cooperative SWIPT NOMA systems”, IEEE Trans. Signal Proces., vol. 65, no. 18, pp. 4874-4886, 2017. DOI: 10.1109/TSP.2017.2715008.

[22] F. Zhou, Z. Li, J. Cheng, Q. Li, and J. Si, "Robust AN-Aided beamforming and power splitting design for secure MISO cognitive radio with SWIPT", IEEE Trans. Wireless Commun., vol. 16, no. 4, pp. 2450-2464, 2017. DOI: 10.1109/TWC.2017.2665465.

[23] H. Wang and X. Xia, "Enhancing wireless secrecy via cooperation: signal design and optimization", IEEE Commun. Magz., vol. 53, no. 12, pp. 47-53, 2015. DOI: 10.1109/MCOM.2015.7355565.

[24] E. Boshkovska, D. W. K. Ng, N. Zlatanov, A. Koelpin, and R. Schober, "Robust resource allocation for MIMO wireless powered communication networks based on a non-linear EH model", IEEE Trans. Commun., vol. 65, no. 5, pp. 1984-1999, 2017. DOI: 10.1109/TCOMM.2017.2664860.

[25] E. Boshkovska, N. Zlatanov, L. Dai, D. W. K. Ng, and R. Schober, "Secure SWIPT networks based on a non-linear energy harvesting model", in Proc. IEEE WCNC 2017, San Francisco, CA, USA, 2017. DOI: 10.1109/WCNCW.2017.7919062.

[26] D. Zwillinger, Table of Integrals, Series, and Products, 7 Ed., Academic Press, 2007. 